\title{
AN APOLOGY
}

\section{som \\ LOLLARD DOCTRINES, \\ ATTRIBUTED TO WICLIFFE.}

\author{
NOW FIRST PRINTED FROM A MANUSCRIPT IN THE LIBRARY \\ OF TRINITY COLLEGE, DUBLIN.
}

WITH AN INTRODUCTION AND NOTES,

BY

JAMES HENTHORN TODD, D.D. V.P.R.I.A.

FELLOW OF TRINITy COLLEgE, AND TREASURER OF ST. PATRICK's CATHEDRAL, DUBLIN.

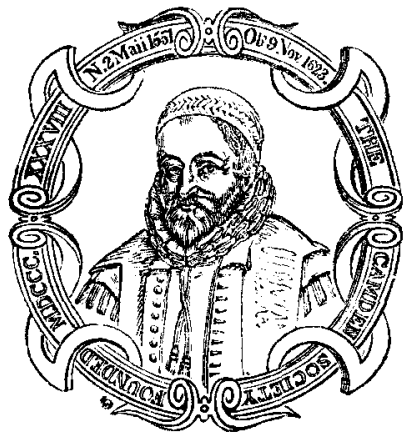

\section{O N D O N :}

PRINTED FOR THE CAMDEN SOCIETY,

BY JOHN BOWYER NICHOLS AND SON, PARLIAMENT STREF'.

M.DCCC.XLII. 
(No, Xx.) 


\section{COUNCIL}

OF

THE CAMDEN SOCIETY,

FOR THE YEAR 1842-3.

\section{President,}

THE RIGHT HON. LORD FRANCIS EGERTON, M.P.

THOMAS AMYOT, ESQ. F.R.S. Treas. S.A. Director. THE RIGHT HON. LORD BRAYBROOKE, F.S.A.

JOHN BRUCE, ESQ. F.S.A. Treasurer.

JOHN PAYNE COLLIER, ESQ. F.S.A.

C. PURTON COOPER, ESQ. Q.C., D.C.L., F.R.S., F.S.A.

T. CROFTON CROKER, ESQ. F.S.A., M.R.I.A.

SIR HENRY ELLIS, K.H., F.R.S., Sec. S.A.

JAMES ORCHARD HALLIWELL, ESQ. F.R.S., F.S.A.

THE REV. JOSEPH HUNTER, F.S.A.

SIR FREDERICK MADDEN, K.H., F.R.S., F.S.A.

JOHN HERMAN MERIVALE, ESQ. F.S.A.

THE REV. LANCELOT SHARPE, M.A., F.S.A.

THOMAS STAPLETON, ESQ. F.S.A.

WILLIAM J. THOMS, ESQ. F.S.A. Secretary.

THOMAS WRIGHT, ESQ. M.A., F.S.A. 